\title{
Mieux vaut prévenir que guérir (pas toujours)
}

\section{Hans Stalder}

Prof. Dr méd., membre de la rédaction

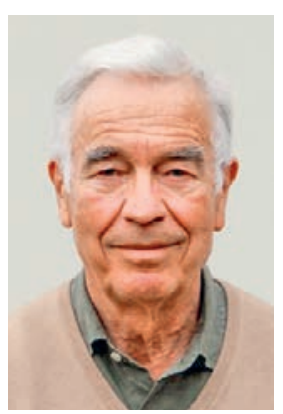

La prévention médicale est certainement une bonne chose et qui serait plus apte à la faire que le médecin? Mais depuis l'échec éclatant de la prescription des hormones chez la femme ménopausée il faut être prudent. L'idée avait une certaine logique: après la ménopause, les hormones féminines diminuent et les femmes vieillissent. Donc pour ralentir le vieillissement il faut remplacer les hormones. Des études de cohorte soutenues par l'industrie et propagées par certains spécialistes semblaient le prouver. Ce ne sont que des études randomisées et prospectives qui, en excluant des cofacteurs tels que des différences socio-économiques, ont pu montrer que les hormones en fait étaient nocives. Ce constat a poussé David Sackett à écrire un éditorial intitulé «The arrogance of preventive medicine» où il dit entre autres: "Without evidence from positive randomized trials (and, better still, systematic reviews of randomized trials) we cannot justify soliciting the well to accept any personal health intervention" (Sackett D. L. The arrogance of preventive medicine. CMAJ. 2002;167:363-4).

Entre-temps d'autres mesures de prévention primaire ont été abandonnées: plus d'aspirine pour tout le monde, plus d'acides gras omega-3, plus de vitamines A et $\mathrm{E}$ et même plus de vitamine $\mathrm{D}$ et calcium chez des personnes âgées...

Et des mesures de dépistage sont aussi mises en question. Le dosage du PSA n'est plus recommandé. La mammographie est contestée. La recherche d'hypothyroïdie, l'examen de routine d'un souffle carotidien ne sont pas indiqués, ni la recherche d'ostéoporose chez tout le monde. Pourquoi avons-nous entrepris toutes ces actions préventives? C'est parce qu'une intervention préventive a l'air séduisante quand les résultats sont présentés par la réduction relative d'un processus pathologique. Par exemple pour l'ostéoporose, où une intervention médicamenteuse a diminué l'incidence de fracture de la hanche de plus de 30\%. Impressionnant? La réduction absolue l'est moins. L'incidence a diminué de 3,5 à 2,6\% après 5 ans [1]. Ce qui donne un nombre de traitements nécessaires (NNT) de 111 pour éviter une fracture. Mais pour le médecin de famille (et en santé publique) ce n'est pas le NNT qui compte uniquement, mais surtout le NNS (number necessary to screen) [2] Pour une incidence d'ostéoporose chez la femme de $15 \%$, le NNS est 6,6 pour dépister un cas. Avec un NNT de 111 ça fait un dépistage à pratiquer chez 733 femmes et de celles-ci 111 à traiter pour éviter une fracture... Pour ces raisons, non seulement le dépistage de l'ostéoporose [3], mais même le dépistage du diabète dans une population sans risque sont contestés [4]. Et savez-vous que la prévention par dépistage du glaucome [5], la prévention du cancer de la peau par des crèmes solaires [6] et la prévention par dépistage des tumeurs de la peau [7] n'ont jamais pu être démontrées par des études sérieuses? Un autre problème est que le dépistage et le traitement préventif qui s'ensuit vont souvent seulement diminuer l'incidence de la maladie testée, mais pas la mortalité totale et ni souvent la morbidité totale. C'est le cas pour le dépistage du cancer du sein par la mammographie et pour la recherche d'anévrisme de l'aorte. Même l'endoscopie ou la recherche de sang dans les selles pour dépister le cancer du côlon ne diminuent pas la mortalité totale. Difficile de dire s'il vaut mieux mourir de cancer ou (à choix) d'infarctus, d'apoplexie ou de démence.

Faut-il donc abandonner la prévention au cabinet? Certes pas! Mais il faut être prudent quand on l'applique à toute la population en tant que prévention primaire (ce qui est plutôt une affaire de politique de santé), sans l'appui de résultats d'études prospectives randomisées. Ceci vaut aussi pour le dépistage. Ce rappel s'adresse particulièrement aux sociétés de spécialistes, afin qu'elles ne soient pas suspectées de prôner de telles mesures par intérêt financier, comme c'est le cas pour l'industrie. Une autre affaire est d'appliquer des mesures préventives secondaires chez des patients à risque. C'est difficile, car il faut personnaliser la prévention. Aidé par une analyse génétique? Pourquoi pas, mais il faudrait alors prouver par des études prospectives randomisées qu'elle est supérieure à une simple anamnèse familiale et personnelle et un bon examen clinique.

Less is more est valable aussi pour la prévention!

Les références se trouvent sous www.bullmed.ch $\rightarrow$ Numéro actuel ou $\rightarrow$ Archives $\rightarrow 2019 \rightarrow 15$. 